\title{
Atomic resolution ptychographic phase contrast imaging of polar-ordered structures in functional oxides
}

Ian MacLaren ${ }^{1}$, Hao Yang ${ }^{2}$, Lewys Jones ${ }^{2}$, Peter D. Nellist ${ }^{2}$, Henning Ryll ${ }^{3}$, Martin Simson ${ }^{4}$, Heike Soltau $^{4}$, Yukihito Kondo ${ }^{5}$, Ryusuke Sagawa ${ }^{5}$, Hiroyuki Banba ${ }^{5}$

1. SUPA School of Physics and Astronomy, University of Glasgow, Glasgow G12 8QQ, UK

2. University of Oxford, Department of Materials, Parks Rd, Oxford, UK

3. PNSensor GmbH, Otto-Hahn-Ring 6, 81739 München, Germany

4. PNDetector GmbH, Sckellstraße 3, 81667 München, Germany

5. JEOL Ltd., 3-1-2 Musashino, Akishima, Tokyo 196-8558, Japan

Scanning transmission electron microscopy (STEM) imaging has traditionally relied on (mostly) incoherent imaging using electrons scattered to high angles [1,2]. This gives strong contrast for imaging heavier atoms, but is only an incomplete technique for in ferroelectrics or polar-ordered functional oxides, since the oxygens are invisible in the presence of the dominant scattering from the heavy cations. In recent years, however, there has been a resurgence in the use of low angle scattered electrons using modes like annular bright field imaging [3] and standard bright field imaging [4,5]. This allows the direct imaging of oxygen atoms, which can be used for a full quantification of the local polarization [5], as has previously been performed using negative $\mathrm{C}_{\mathrm{S}}$ imaging using high resolution TEM [6].

In principle, however, it is possible to reconstruct the phase at atomic resolution using ptychographic methods from suitable diffraction patterns with overlapping diffraction discs recorded at each scan point in a STEM image [7]. However, to do so practically requires the rapid recording of diffraction data, both to allow the acquisition of images with reasonable numbers of pixels in a sensible time scale, as well as to minimize the distortion images in real-space due to sample-drift. We show that this has now become possible by exploiting a direct electron detector running at 1000 frames per second (fps) or greater, which is a massive improvement over earlier CCDs running at $<30 \mathrm{fps}$.

This was applied to an unusual antiphase boundary in $\mathrm{Nd}$, $\mathrm{Ti}$ codoped $\mathrm{BiFeO}_{3}$, which has been wellcharacterised previously by conventional atomic resolution STEM [8,9] and HRTEM techniques. The atomic structure and chemistry of this boundary is consequently well-understood making this an ideal test object for atomic resolution ptychography. Atomic resolution datasets were recorded using a JEOL ARM200F, equipped with a cold FEG source and fitted with a PNDetector pnCCD (S)TEM camera running at up to $2000 \mathrm{fps}$. Reconstructions were performed using algorithms published previously [7].

The resulting phase image is shown in Figure 1 (top) and is compared to the HAADF image acquired simultaneously. The phase image shows a contrast similar to that previously seen in negative $\mathrm{C}_{\mathrm{S}}$ images of the same structure and can be assigned to atoms by comparison with the HAADF image. The strong red peaks in the phase image are coincident with the green peaks in the HAADF image and must be mixed B-site (Fe/Ti) / O columns. The broad yellow/orange peaks in the phase image are coincident with the bright red peaks in the HAADF image and must be A-site $(\mathrm{Bi} / \mathrm{Nd})$ columns. Finally, the weaker yellow peaks in the phase image are not seen in the HAADF image, but sit in positions which would fit expectations for pure O columns. It will clearly be possible to determine the atom positions quantitatively from such images and therefore perform atomic resolution polarization calculations, which will be reported at the conference and compared with the results calculated from atomic 
resolution BF/HAADF STEM and negative $\mathrm{C}_{\mathrm{S}}$ TEM imaging. It is nevertheless, clearly demonstrated that atomic resolution ptychography can be added to the roster of techniques for quantitative materials characterization at the atomic scale, provided suitable detectors are used.

\section{References:}

[1] AV Crewe, J Wall and LM Welter: J. Appl. Phys. 39 (1968) p. 5861.

[2] P Hartel, H Rose and C Dinges: Ultramicroscopy 63 (1996) pp. 93-114.

[3] M Hammel and H Rose: Ultramicroscopy 58 (1995) pp. 403-415.

[4] JM LeBeau et al., Phys. Rev. B, 80 (2009) 174106.

[5] I MacLaren, et al., APL Mater. 1 (2013) 021102.

[6] CL Jia et al., Nature Mater., 7 (2008) pp. 57-61.

[7] TJ Pennycook et al., Ultramicroscopy (2015) in press (doi:10.1016/j.ultramic.2014.09.013).

[8] I MacLaren et al., APL Materials, 1 (2013) 021102.

[9] I MacLaren et al., APL Materials, 2 (2014) 066106

[10] The authors gratefully acknowledge funding from the EPSRC under grant numbers EP/M009963/1 and EP/M010708/1. This work was supported by the EU FP7 Grant Agreement 312483 (ESTEEM2).

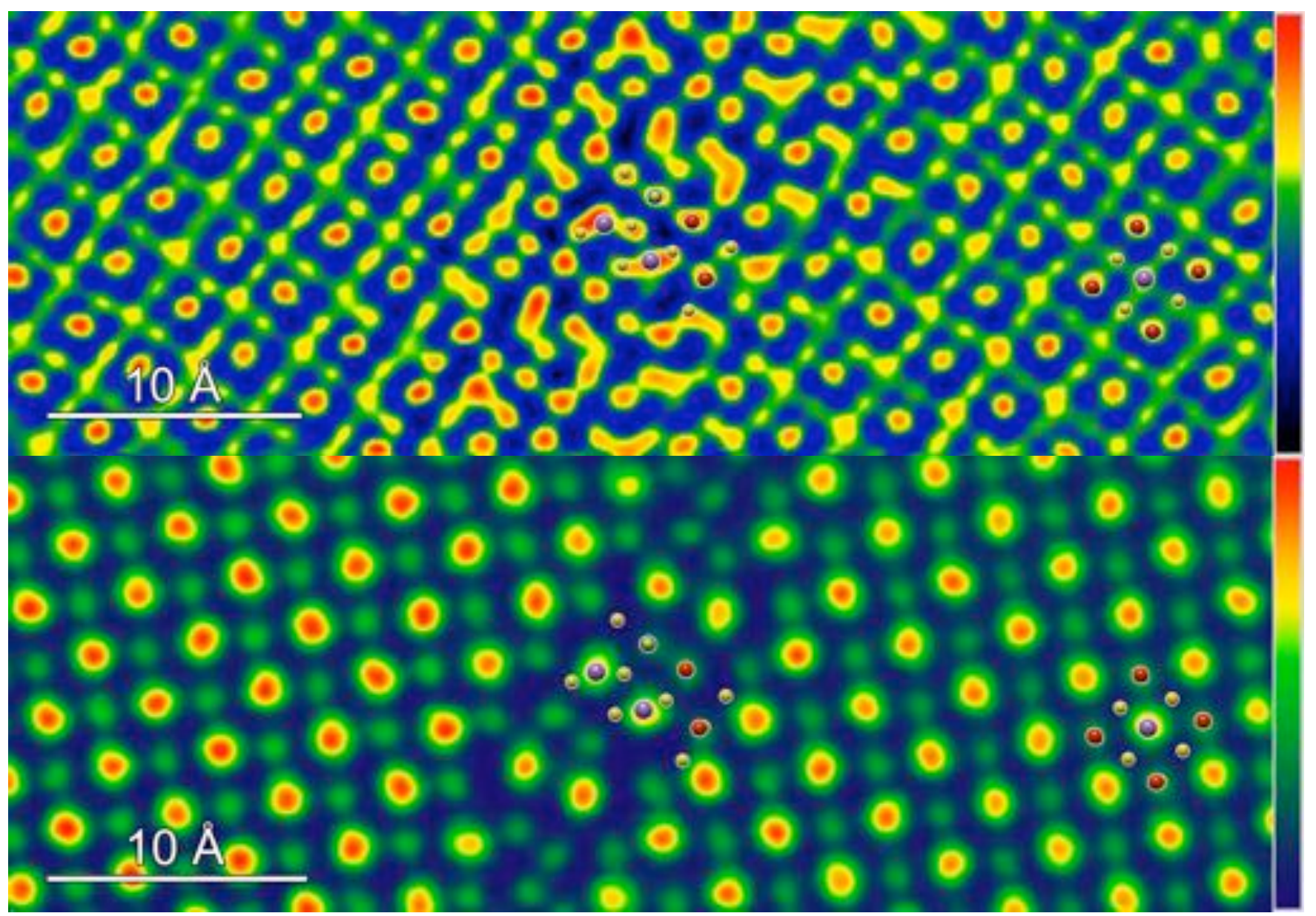

Figure 1. (top) Reconstructed phase image of the boundary. (bottom) HAADF image recorded simultaneously with the diffraction dataset, but independent from the calculation of the phase image. Atom column positions are overlaid (red - iron, purple - bismuth, blue - titanium, yellow - oxygen). 